J. Clin. Chem. Clin. Biochem.

Vol. 15,1977 , pp. $485-488$

\title{
Enzymdiagnostik in lipämischen Seren vor und nach Polyanionenpräzipitation mit Heparin und Magnesiumchlorid
}

\author{
Von J. Freise, P. Magerstedt und Ellen Schmidt \\ Abteilung für Gastroenterologie und Hepatologie (Leiter: Prof. Dr. med. F. W. Schmidt) im Department für Innere \\ Medizin der Medizinischen Hochschule Hannover
}

(Eingegangen am 28. Dezember 1976/6. April 1977)

\begin{abstract}
Zusammenfassung: Es wird kritisch zu der Methode der Enzymaktivitätsbestimmung in trüben, lipämischen Seren nach Polyanionenpräzipitation mit Heparin und Magnesiumchlorid Stellung genommen. Es zeigt sich, daß gerade bei der Beurteilung von Leber- und Pankreaserkrankungen, die häufig mit Hyperlipoproteinämien verbunden sind, die Polyanionenpräzipitation nur noch zu Restaktivitäten im geklärten Serum führt. Bei der Glutamatdehydrogenase und der $\alpha$-Amylase im Phadebastest kommt es durch Heparin und Magnesiumchlorid zu einer Inaktivierung der Enzyme, während bei der $\gamma$-Glutamyltransferase die erniedrigte Enzymaktivität im geklärten Serum dadurch verursacht wird, daß die $\gamma$-Glutamyltransferase mit den Lipoproteinen, am ehesten mit Chylomikronen, durch die Polyanionenpräzipitation in den lipidreichen Uberstand überführt wird.
\end{abstract}

Auch die saure Phosphatase zeigt im geklärten Serum nur noch Restaktivitäten.

Die Aktivität von Aspartataminotransferase, Alaninaminotransferase, alkalischer Phosphatase, Leucinarylamidase, Cholinesterase, Creatinkinase, Lactatdehydrogenase und $\alpha$-Hydroxybutyratdehydrogenase und die $\alpha$-Amylase im Merckotest bleibt durch die Polyanionenpräzipitation unbeeinflußt.

\section{Enzyme diagnosis in lipaemic sera before and after polyanion precipitation with heparin and magnesium chloride}

Summary: The method for the determination of enzymic activity in turbid, lipaemic sera, which involves clearing by polyanion precipitation with heparin and magnesium chloride, was critically reviewed. In the diagnosis of diseases of the liver and pancreas, which are frequently associated with hyperlipoproteinaemia, only residual enzyme activities are measured in the cleared serum after polyanion treatment. In the measurement of glutamate dehydrogenase and in the Phadebas test for $\alpha$-amylase, the enzymes are inactivated by treatment with heparin and magnesium chloride. On the other hand, as a result of polyanion precipitation $\gamma$-glutamyl transferase is transferred, together with lipoproteins and chylomicrons, to the lipid-rich supernatant. Acid phosphatase also exhibits only residual activity in cleared serum. The activity of aspartate aminotransferase, alanine aminotransferase, alkaline phosphatase, leucine arylamidase, cholinesterase, creatine kinase, lactate dehydrogenase, and $\alpha$-hydroxybutyrate dehydrogenase, and the activity of $\alpha$-amylase in the Merckotest are not affected by polyanion treatment of the serum.

\section{Einleitung}

Die Bestimmung von Enzymaktivitäten kann, wie alle photometrischen Messungen in trüben, d.h. vor allem in lipämischen Seren erheblich gestört sein. Es ist daher üblich (1), durch Polyanionenpräzipitation mit Heparin und Magneșiumchlorid die triglyceridreichen Lipoproteine, die die Trübung von Șeren meist verursachen, zu fällen und sie durch Zentrifugation in einen lipidreichen Ubberstand zu überführen, der verworfen wird.
In so ,geklärten“ Seren lassen sich Bestimmungen von Enzymaktivitäten ohne Schwierigkeiten durchführen.

Im Gegensatz zu den Ergebnissen von Lambrecht \& Seidel (1) machten wir die Erfahrung, daß bei der $\gamma$-Glutamyltransferase die Aktivität im geklärten Serum niedriger ist als im trüben lipämischen Serum. Wir haben in zwei früheren Publikationen $(2,3)$ an Einzelfällen 
zeigen können, daß die $\gamma$-Glutamyltransferase sich an Chylomikronen anlagert und bei der Präzipitation mit Heparin und Magnesiumchlorid mit diesen in den lipidreichen Uberstand übergeführt wird.

Bei der Bestimmung von Enzymaktivitäten in mit Heparin und Magnesiumchlorid ,geklärten " Seren geht man von der Annahme aus, daß sich die Enzymaktivität durch die Polyanionenpräzipitation nicht ändert, und daß sich vor allem kein Enzym im lipämischen Uberstand anreichert. Diese Annahme hat sich jedoch für die $\gamma$-Glutamyltransferase als falsch erwiesen $(2,3)$.

Es galt nun zu untersuchen, ob das Verhalten der $\gamma$ Glutamyltransferase bei der Polyanionenpräzipitation eine Ausnahme darstellt, oder ob auch andere der im Routinegebrauch bestimmten Enzymaktivitäten niedrigere Werte nach der Präzipitation der Lipoproteine ergeben als im lipämischen Serum. Für unsere Untersuchungen haben wir lipämische Seren verwandt, die zwar sehr trübe waren, aber nicht so milchig, daß eine Enżymbestimmung im nativen Serum nicht möglich war.

Wir haben in klaren und trüben Seren vor und nach Zugabe von Heparin und Magnesiumchlorid neben dem Cholesterin- und Triglyceridgehalt folgende Enzymaktivitäten bestimmt:

Aspartataminotransferase (EC 2.6.1.1)

Alaninaminotransferase (EC 2.6.1.2)

Glutamatdehydrogenase (EC 1.4.1.3)

Alkalische Phosphatase (EC 3.1.3.1)

Leucinarylamidase (EC 3.4.11)

$\gamma$-Glutamyltransferase (EC 2.3.2.2)

Cholinesterase (EC 3.1.1.8)

Creatinkinase (EC 2.7.3.2)

Lactatdehydrogenase (EC 1.1.1.27)

$\alpha$-Hydroxybutyratdehydrogenase

$\alpha$-Amylase (EC 3.2.1.1)

saure Phosphatase (EC 3.1.3.2)

\section{Material und Methoden}

Heparin-Magnesiumchloridlösung:

Heparinlösung: Thrombophob ad inj. $5000 \mathrm{IE} / \mathrm{ml}$ HeparinNatrium (Fa. NORDMARK Werke)

Magnesiumlösung: $2 \mathrm{~mol} / \mathrm{l} \mathrm{MgCl} 2$ p.a. (Fa. MERCK, Nr. 5833)

Präzipitationstechnik:

9 Teilen Serum wurde 1 Teil einer Mischung von gleichen Volumina Heparinlösung und $\mathrm{MgCl}_{2}$-Lösung zugesetzt. Nach Mischen und 30 minütigem Stehen bei Raumtemperatur wurde $10 \mathrm{~min}$ bei $10000 \mathrm{~g}$ zentrifugiert. Das lipidreiche Präzipitat an der Oberfläche wurde mit EPPENDORF-Pipetten abgehoben. Im trüben und geklärten Serum wurden dann die Enzymaktivitäten parallel bestimmt.

\section{Enzymbestimmungen}

Aspartataminotransferase:

Optimierter Monotest der Fa. BOEHRINGER,

Alaninaminotransferase: Mannheim, Nr. 124362

Optimierter Monotest der Fa. BOEHRINGER, Mannheim, Nr. 124976
GJutamatdehydrogenase:

Optimierter, aktivierter Monotest der Fa. BOEHRINGER, Mannheim, Nr. 124311

Creatinkinase:

Aktivierter Monotest der Fa. BOEHRINGER, Mannheim, Nr. 124150

Lactatdehydrogenase:

Testkombination der Fa. BOEHRINGER, Mannheim, Nr. 124893

$\alpha$-Hydroxybutyratdehydrogenase:

Testkombination der Fa. BOEHRINGER,

Saure Phosphatase: Mannheim, Nr. 124818

Alkalische Phosphatase:

Testkombination der Fa. BOEHRINGER, Mannheim, Nr. 125008

Leucinarylamidase:

Merckotest, Fa. MERCK, Darmstadt, Nr. 3314

$\gamma$-Glutamyltransferase:

Merckotest, Fa. MERCK, Darmstadt, Nr. 3359

Monotest der Fa. BOEHRINGER, Mannheim, Nr. 125631

Cholinesterase:

Testkombination (Substrat: Acetylthiocholin) der Fa. BOEHRINGER, Mannheim, Nr. 124117

$\alpha$-Amylase:

(MERCK)

Merckotest, Fa. MERCK, Darmstadt, Nr. 3301

Phadebas $\alpha$-Amylase-Test der Fa. PHARMACIA

\section{Lipidbestimmungen}

Cholesterin: Testkombination (Enzymatischer Farbtest) der Fa. BOEHRINGER, Mannheim, Nr. 124087

Triglyceride: Testkombination der Fa. BOEHRINGER, Mannheim, Nr. 124032

Untersuchungsmaterial

Das klare und das trübe Serum mit z.T. erheblich erhöhten Enzymaktivitäten wurde nach Punktion der Cubitalvene von einem unausgewählten Krankengut der Medizinischen Hochschule Hannover gewonnen.

\section{Ergebnisse}

In einer ersten Testserie wurde der Einfluß der Heparin$\mathrm{MgCl}_{2}$-Lösung auf die Enzymbestimmung im klaren Serum untersucht. Die Ergebnisse sind in Tabelle 1 a dargestellt und zeigen, daß die Enzymbestimmungen von Aspartataminotransferase, Alaninaminotransferase, Creatinkinase, Lactatdehydrogenase, $\alpha$-Hydroxybutyratdehydrogenase, alkalischer Phosphatase, Leucinarylamidase, $\gamma$-Glutamyltransferase, Cholinesterase und $\alpha$-Amylase (MERCK) durch Heparin und $\mathrm{MgCl}_{2}$ unbeeinflußt bleiben.

Inkonstante Restaktivitäten zeigen sich bei der Glutamatdehydrogenase, der $\alpha$-Amylase (Phadebas) und der sauren Phosphatase. Der Cholesterin- und Triglyceridgehalt änderte sich nach Zusatz von Heparin und $\mathrm{MgCl}_{2}$ nicht.

In der zweiten Testserie wurden die Enzyme, die in der ersten Testserie unbeeinflußt geblieben waren, in lipämischen, trüben Seren vor und nach Präzipitation åuf Aktivitätsänderungen überprüft (Tab. 1 b). Bei allen Enzymen bis auf die $\gamma$-Glutamyltransferase waren im t-Test für gebundene Stichproben vor und nach Präzi- 
Tab. 1. Katalytische Konzentrationen der untersuchten Enzyme und Lipidgehalt

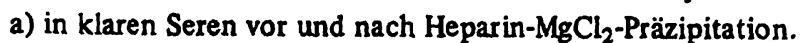

b) in lipämischen Seren vor und nach Heparin- $\mathrm{MgCl}_{2}-$ Präzipitation.

$N=$ Anzahl der untersuchten Seren

$\overline{\mathbf{x}}=$ Mittelwerte der Enzy maktivitäten in $\mathrm{U} / \mathrm{l}$ vor Heparin- $\mathrm{MgCl}_{2}-\mathrm{Präzipitation}$

$\overline{\mathrm{y}}=$ Mittelwerte der Enzymaktivitäten in $\mathrm{U} / 1$ nach Heparin- $\mathrm{MgCl}_{2}-\mathrm{Präzipitation}$

$d=$ Differenz der Mittelwerte

$S=$ Signifikanz im t-Test für gebundene Stichproben. n.s. = nicht signifikant

Bereich: Seren mit der niedrigsten bzw. höchsten katalytischen Konzentration

Tab. 1 a

\begin{tabular}{|c|c|c|c|c|c|c|}
\hline & $\mathbf{N}$ & $\begin{array}{l}\text { Bereich } \\
{[U / 1]}\end{array}$ & $\begin{array}{l}\bar{x} \\
{[U / 1]}\end{array}$ & $\begin{array}{l}\bar{y} \\
{[U / 1]}\end{array}$ & $\begin{array}{l}d \\
{[U / 1]}\end{array}$ & $S$ \\
\hline $\begin{array}{l}\text { Aspartataminotransferase } \\
\text { Alaninaminotransferase } \\
\text { Glutamatdehydrogenase } \\
\text { Creatinkinase } \\
\text { Lactatdehydrogenase } \\
\alpha \text {-Hydroxybutyratdehydrogenase } \\
\text { Saure Phosphatase } \\
\text { Alkalische Phosphatase } \\
\text { Leucinarylamidase } \\
\gamma \text {-Glutamyltransferase } \\
\text { Cholinesterase } \\
\alpha \text {-Amylase (MERCK) } \\
\alpha \text {-Amylase (Phadebas) }\end{array}$ & $\begin{array}{r}10 \\
10 \\
14 \\
10 \\
10 \\
10 \\
6 \\
10 \\
16 \\
10 \\
10 \\
16 \\
7\end{array}$ & $\begin{array}{r}7-273 \\
7-490 \\
1-113 \\
4-176 \\
105-304 \\
55-117 \\
2-\quad 4 \\
92-1529 \\
11-151 \\
9-500 \\
1067-3537 \\
93-316 \\
165-335 \\
{[\mathrm{mmol} / 1]}\end{array}$ & $\begin{array}{r}52 \\
105 \\
21 \\
51 \\
198 \\
91 \\
3 \\
319 \\
50 \\
179 \\
2325 \\
176 \\
231 \\
\\
{[\mathrm{mmol} / \mathrm{l}]}\end{array}$ & $\begin{array}{r}54 \\
103 \\
11 \\
48 \\
185 \\
90 \\
2 \\
328 \\
46 \\
169 \\
2383 \\
170 \\
39 \\
\\
{[\mathrm{mmol} / 1]}\end{array}$ & $\begin{array}{r}2 \\
2 \\
10 \\
3 \\
13 \\
1 \\
1 \\
9 \\
4 \\
10 \\
58 \\
6 \\
192 \\
{[\mathrm{mmol} / \mathrm{l}]}\end{array}$ & $\begin{array}{l}\text { n.s. } \\
\text { n.s. } \\
\mathrm{P}<0.02 \\
\text { n.s. } \\
\text { n.s. } \\
\text { n.s. } \\
\mathrm{P}<0.001 \\
\text { n.s. } \\
\text { n.s. } \\
\text { n.s. } \\
\text { n.s. } \\
\text { n.s. } \\
P<0.001\end{array}$ \\
\hline $\begin{array}{l}\text { Cholesterin } \\
\text { Triglyceride }\end{array}$ & $\begin{array}{r}10 \\
8\end{array}$ & $\begin{array}{ll}4- & 8 \\
1- & 2\end{array}$ & $\begin{array}{l}5 \\
1\end{array}$ & $\begin{array}{l}5 \\
1\end{array}$ & $\begin{array}{l}0 \\
0\end{array}$ & $\begin{array}{l}\text { n.s. } \\
\text { n.s. }\end{array}$ \\
\hline
\end{tabular}

Tab. 1 b.

\begin{tabular}{|c|c|c|c|c|c|c|}
\hline & $\mathbf{N}$ & $\begin{array}{l}\text { Bereich } \\
{[U / 1]}\end{array}$ & $\begin{array}{l}\bar{x} \\
{[U / 1]}\end{array}$ & $\begin{array}{l}\bar{y} \\
{[U / 1]}\end{array}$ & $\begin{array}{l}d \\
{[U / 1]}\end{array}$ & $S$ \\
\hline $\begin{array}{l}\text { Aspartataminotransferase } \\
\text { Alaninaminotransferase } \\
\text { Creatinkinase } \\
\text { Lactatdehydrogenase } \\
\alpha \text {-Hydroxybutyratdehydrogenase } \\
\text { Alkalische Phosphatase } \\
\text { Leucinarylamidase } \\
\gamma \text {-Glutamyltransferase } \\
\text { Cholinesterase } \\
\alpha \text {-Amylase (MERCK) }\end{array}$ & $\begin{array}{r}10 \\
6 \\
13 \\
22 \\
22 \\
23 \\
19 \\
22 \\
23 \\
12\end{array}$ & $\begin{array}{r}7-45 \\
8-25 \\
6-121 \\
79-275 \\
46-136 \\
68-2460 \\
14-276 \\
3-1450 \\
774-5428 \\
123-483 \\
{[\mathrm{mmol} / \mathrm{l}]}\end{array}$ & $\begin{array}{r}20 \\
14 \\
27 \\
148 \\
90 \\
500 \\
53 \\
424 \\
2358 \\
243 \\
{[\mathrm{mmol} / \mathrm{l}]}\end{array}$ & $\begin{array}{r}21 \\
15 \\
27 \\
146 \\
84 \\
484 \\
54 \\
309 \\
2324 \\
253 \\
{[\mathrm{mmol} / \mathrm{l}]}\end{array}$ & $\begin{array}{r}1 \\
1 \\
0 \\
2 \\
6 \\
16 \\
1 \\
115 \\
34 \\
10 \\
\end{array}$ & $\begin{array}{l}\text { n.s. } \\
\text { n.s. } \\
\text { n.s. } \\
\text { n.s. } \\
\text { n.s. } \\
\text { n.s. } \\
\text { n.s. } \\
\text { P }<0.01 \\
\text { n.s. } \\
\text { n.s. }\end{array}$ \\
\hline $\begin{array}{l}\text { Cholesterin } \\
\text { Triglyceride }\end{array}$ & $\begin{array}{l}19 \\
21\end{array}$ & $\begin{array}{l}1-19 \\
2-\quad 13\end{array}$ & $\begin{array}{l}11 \\
6\end{array}$ & $\begin{array}{l}4 \\
3\end{array}$ & $\begin{array}{l}7 \\
3\end{array}$ & $\begin{array}{l}P<0.001 \\
P<0.001\end{array}$ \\
\hline
\end{tabular}

pitation keine signifikanten Unterschiede der Enzymaktivitäten erkennbar. Der Verdünnungsfaktor von $1: 10$ bei den kḷaren und lipämischen Seren ist bei der Berechnung berücksichtigt worden. Die Aktivität der $\gamma$-Glutamyltransferase war jedoch im trüben lipämịschen Serum im Mittel um 37,2\% höher als im geklärten Serum.

In 11 weiteren Seren wurde anschließend die $\gamma$ Glutamyltransferase-Aktivität im lipämischen Serum, im geklärten Serum und im lipidreichen Uberstand nàch Präzịițation bestimmt. Die katalytische Konzentration von $\gamma$-Glutamyltransferase im lipämischen Serum betrug in diesen 11 Seren im Durchschnitt $931 \mathrm{U} / \mathrm{l}$, im geklärten Serum 691 U/1 und im lipidreichen präzipitierten Überstand 1568 U/1. Die Unterschiede (Tab. 2) waren im $\mathrm{t}$-Test für gebundene Stichproben hochsignifikant $(P<0,001)$.

\section{Diskussion}

Die Ergebnisse unserer ersten Versuchsreihe mit klaren Seren zeigen, daß Heparin und $\mathrm{MgCl}_{2}$ in der angegebenen Konzentration die Enzymbestimmung von Aspartataminotransferase, Alaninaminotransferase, Creatinkinase, Lactatdehydrogenase, $\alpha$-Hydroxybutyratdehydrogenase, alkalischer Phosphatase, Lactatdehydrogenase, $\gamma$-Glut- 
Tab. 2. Vergleich der katalytischen Konzentration von $\gamma$-Glutamyltransferase im trüben Serum $(\overline{\mathbf{x}})$, geklärten Serum $(\bar{y})$ und lipidreichen Uberstand (z).

$\mathrm{N}=$ Anzahl der untersuchten Seren

Bereich: Seren mit der niedrigsten bzw. höchsten katalytischen Konzentration

$\mathrm{S}=$ Signifikanz im t-Test für gebundene Stichproben

Die Unterschiede der $\gamma$-Glutamyltransferase-Aktivität sind hochṣignifikant

\begin{tabular}{llllllll}
\hline & $N$ & $\begin{array}{l}\text { Bereich } \\
{[\mathrm{U} / 1]}\end{array}$ & $\begin{array}{l}\overline{\mathrm{x}} \\
{[\mathrm{U} / 1]}\end{array}$ & $\begin{array}{l}\overline{\mathrm{y}} \\
{[\mathrm{U} / 1]}\end{array}$ & $\begin{array}{l}\overline{\mathrm{z}} \\
{[\mathrm{U} / \mathrm{l}]}\end{array}$ & $\mathrm{S}_{\mathbf{x y}}$ & $\mathrm{S}_{\mathbf{x z}}$ \\
\hline$\gamma$-Glutamyltransferase & 11 & $199-1630$ & 931 & 691 & 1568 & $\mathrm{P}<0.001$ & $\mathrm{P}<0.001$ \\
\hline
\end{tabular}

amyltransferase, Cholinesterase und $\alpha$-Amylase im Merckotest nicht stören. Bei der Glutamatdehydrogenase, der $\alpha$-Amylase im Phadebas-Test und bei der sauren Phosphatase können nach Heparin- und $\mathrm{MgCl}_{2}$-Zusatz nur noch Restaktivitäten gemessen werden.

Der Mechanismus der Glutamatdehydrogenase-Hemmung durch Heparin ist seit den Arbeiten von Horn \& Bruns (4) bekannt und findet hier nur eine Bestätigung. Auch bei der sauren Phosphatase und der $\alpha$-Amylase im Phadebastest ist die Aktivitätshemmung durch Heparin und $\mathrm{MgCl}_{2}$ schon von Lambrecht \& Seidel (1) beschrieben und z.T. erklärt worden.

Die Polyanionenpräzipitation mit Heparin und $\mathrm{MgCl}_{2}$ ist somit nicht geeignet zur Klärung von Seren, in denen Glutamatdehydrogenase, saure Phosphatase und $\alpha$ Amylase im Phadebastest bestimmt werden sollen. $\mathrm{Da}$ die Heparin- $\mathrm{MgCl}_{2}$-Lösung nicht $\mathrm{zu}$ einer Inhibition der $\alpha$-Amylase führt - siehe Ergebnisse im Merckotest dürfte es im Phadebastest am ehesten zu einer Beeinflussung aller freigesetzten Chromophore durch die Heparin- $\mathrm{MgCl}_{2}$-Lösung kommen.

Die Cholesterin- und Tryglyceridkonzentration wird bei klaren Seren durch die Polyanionenpräzipitation nicht verändert.

\section{Literatur}

1. Lambrecht, J. \& Seidel, D. (1974), J. Clin. Chem. Clin. Biochem. 12, 154-158.

2. Freise, J., Magerstedt, P. \& Schmidt, E. (1976), J. Clin. Chem. Clin. Biochem. 14, 589-594.
In der żweiten Versuchsreihe mit lipämischen Seren war bei allen gemessenen Enzymen bis auf die $\gamma$-Glutamyltransferäse im t-Test für gebundene Stichproben vor und nach Präzipitation kein signifikanter Unterschied der Enzymaktivitäten erkennbar.

Die Aktivität der $\gamma$-Glutamyltransferase war jedoch im trüben, lipämischen Serum im Mittel um 37\% höher als im geklärten Serum. Die Ursache für die erniedrigte $\gamma$-Glutamyltransferase-Aktivität im geklärten Serum ist darin begründet, daß die $\gamma$-Glutamyltransferase sich mit den Fettpartikeln bei der Präzipitation im lipidreichen Überstand anreichert.

Zusammenfassend ergeben unsere Versuche, daß nach einer Polyanionenpräzipitation mit Heparin und $\mathrm{MgCl}_{2}$ im geklärten Serum von den im Medizinischen Labor häufig bestimmten Enzymen nur folgende Enzymaktivitäten unbeeinflußt sind:

Aspartataminotransferase, Alaninaminotransferase, Creatinkinase, Lactatdehydrogenase, $\alpha$-Hydroxybutyratdehydrogenase, alkalische Phosphatase, Leucinarylamidase, Cholinesterase und die $\alpha$-Amylase im Merckotest.

Die Bestimmung der $\gamma$-Glutamyltransferase-Aktivität muß im Nativ-Serum erfolgen.

3. Freise, J., Magerstedt, P. \& Schmidt, E. (1976), Clin. Chim. Acta 73, 267-275.

4. Horn, H. D. \& Bruns, F. H. (1958), Biochem. Z. 331, 58-64.

Dr. med. Jürgen Freise

Medizinische Hochschule Hannovẹ

Abteilung für Gastroenterologie

und Hepatologie

Karl-Wiechert-Allee 9

3000 Hannover 61 